\title{
Video Tutorials in Academic Art Libraries: A Content Analysis and Review
}

Eamon Tewell

Combining the informative nature of library tutorials with the interactivity of online video, video tutorials and screencasts have become recognized as an effective way to provide instruction in an engaging manner. The visual nature of video makes the medium highly appropriate at institutions serving visual arts researchers, who regularly seek images for edification. This article examines the availability and quality of video tutorials among 290 academic libraries serving arts students, finding that 48 percent of institutions include instructional videos on their Web sites. Increased tutorial development on diverse topics using varied recording methods is recommended based on the results.

\section{Introduction}

Libraries have long realized the necessity of meeting user needs online, and for years have worked to make print collections, visual resources, and reference services accessible in a digital environment. At many institutions the reach of services being offered continues to expand even as budgets contract. Academic art libraries present users with an array of learning opportunities, including classroom instruction, individual consultations, and reference desk assistance. One significant instructional development within the last decade is that of online tutorials, which meet the needs of library users when and where they seek support. Online tutorials have evolved from static Web pages to multimedia experiences. With many users visiting their libraries' Web sites regularly, video tutorials represent a unique way to interact with patrons. The use of online tutorials for library instruction is on the rise, as indicated by increasingly frequent discussion via e-mail listservs, at conferences, and in the library literature. The widespread popularity of video is due in large part to the rich user experience it offers compared to text and static images.

The increasing discussion and presence of video tutorials may be attributed to the unique advantages they offer: online point-of-need assistance to students, asynchronous instruction to distance learners, and meeting the interactive, technology-based learning preferences of the Millennial Generation. Online tutorials also have the ability to reach more users than traditional in-person instruction, providing instant access to instruction on research skills, electronic resources, or any topic the library desires. Most importantly, online videos meet the needs of students who spend an increasing amount of time online and are comfortable using multimedia, frequently preferring it to text-based learning. Meeting patron expectations is a key reason to offer online video. Students expect video from the Web sites they visit, and video tutorials supply them with convenient, concise information.

An additional factor in the adoption of online tutorials is that the software used to create tutorials has become increas- ingly effective and user friendly, no longer requiring knowledge of advanced programming code to produce a polished video. Screencasting and video editing software makes it relatively simple to develop and publish online instruction. Video tutorials also expand the capacity of limited staff resources, allowing librarians to save time by creating a learning module once and making it available online for users to view at any time, as often as they like. With frequently asked questions in particular, Web tutorials reduce the time-consuming demands of in-person instruction. Prerecorded instructional videos remain accessible on the library Web site, providing information as needed.

A compelling reason for art libraries to develop video tutorials stems from the learners being served. Many art librarians recognize the importance of visual learning. Whether for inspiration or reference, images are of major importance to arts researchers, and libraries must supply additional opportunities for visual learning. Video tutorials engage Web site visitors as well as contribute to effective online learning. Additionally, an increasing number of academic libraries supply audio-visual equipment such as video cameras for students and faculty, thereby making the expensive equipment required to produce online videos readily available. ${ }^{1}$ Art libraries in particular may be able to benefit from the expertise of student workers who have experience creating multimedia content.

This study makes a distinction between static Web page tutorials and video tutorials. The latter may consist of a video, screencast, or animation, while the former is most often a series of text-oriented Web pages. Until recently, libraries have created HTML Web pages linked together as a tutorial. These tutorials have progressed towards increased interactivity, incorporating Flash, improved graphics, and sound in an attempt to appeal to different learning styles. Software programs have made the process of creating a static Web tutorial, requiring knowledge of HTML or Flash, into a process as uncomplicated as recording one's computer screen, and with much more effective results for the learner. The term "online tutorial" frequently refers to both HTML and video tutorials. While the two share key char- 
acteristics and the intent to inform users, videos have a wider array of uses, including promotion, marketing, and entertainment. HTML tutorials are primarily designed to instruct, while library videos are just as likely to be as amusing as they are informative.

In many ways, video tutorials are the learning tool toward which static Web page tutorials have been evolving. Examples of video tutorials include a tour of one's library, a screencast demonstration of advanced searching in the Avery Index, or a stop-motion animated video showcasing a periodicals collection. Screencasting and video recording software are relatively simple to learn, making online instruction within the reach of more libraries than ever before. Screencasting in particular has an easy learning curve, allowing the creator to edit, narrate, and publish any task performed on a computer as a video. The end result is a learning object that is designed to instruct, inform, or engage library users.

\section{Purpose of Study}

The purpose of this study is to identify and evaluate video tutorials created by academic libraries serving students in the visual arts, as well as to serve as an introductory analysis of a digital medium that will become an increasingly important learning tool at academic art libraries. It takes stock of tutorials in an attempt to determine whether academic art libraries are taking advantage of online instruction opportunities, while also suggesting directions for future video-based initiatives.

By surveying the availability of video tutorials, evaluating their quality, and identifying commonalities among existing tutorials, the author seeks to answer the following questions:

- Are academic and art school libraries offering instructional videos?

- What types of video tutorials are being offered?

- What is the level of quality of these tutorials?

Numerous studies have compared the efficacy of online and in-person instruction; this article addresses tutorials from a different perspective by evaluating the presence and comparative quality of existing video-learning objects. Research in the area of online tutorials and student learning outcomes presents conflicting results. Some authors found online tutorials to be the most effective in accomplishing learning objectives, others recommended face-to-face instruction, and a majority of studies found no difference between online and in-person instruction. ${ }^{2}$ A comprehensive literature review by Li Zhang, Erin Watson, and Laura Banfield demonstrated that online and face-to-face approaches are equally successful. ${ }^{3}$ Comparing a multimedia tutorial with a static Web tutorial, Nadaleen Tempelman-Kluit found that the screencast tutorial contained more components aiding knowledge retention and was therefore more likely to result in successful learning outcomes. ${ }^{4}$

For clarity, the key terms are defined as follows:

- Academic or Art School Library: A library affiliated with an institution of higher education supporting the study of the visual arts.

- Tutorial: A Web page or video created for instructional purposes, such as demonstrating the use of a database, or for promotional and marketing purposes, such as a library tour.

- Video: A live video, screencast, or animation hosted on the library's Web site or a video-sharing Web site such as YouTube.

\section{Literature Review}

An extensive body of research regarding online tutorials exists, yet studies surveying the existence and quality of video tutorials, particularly in art libraries, are absent. The subject of video as a medium for presenting library information is not a new one. Analog video as an instructional tool first made an appearance in the literature in 1980, with John Lolley's survey of academic library usage of video in instruction sessions. ${ }^{5}$ An abundance of writing on the topic of video in libraries soon followed. Librarians at Iowa State University found improved user attitudes towards the library after integrating video with class instruction. ${ }^{6}$ Other university librarians incorporated video into required bibliographic instruction courses with favorable patron feedback. ${ }^{7}$ Jean Smith's article expressed the need for providing instruction using the same video technology with which students are comfortable, and described videos produced by academic libraries to engage students in the research process. ${ }^{8}$ Smith argued for instruction that enables students to learn on their own terms by using the methods most suited to their interests-an assertion highly applicable to today's digital video tutorials. James Shedlock and Edward Tawyea detailed their use of video for a medical library's orientation and provided best practices for planning videotape production. ${ }^{9}$ Analog video as a means to engage and instruct users is also the subject of articles by Ann Taylor Blauer ${ }^{10}$ and Daren Callahan, ${ }^{11}$ both of whom emphasized the impact of video as a medium to market various library services, particularly as a means to extend one's reach among a broader population of students. Surveying a large, primarily freshman population, Eileen Wakiji and Joy Thomas found a correlation between the viewing of a brief library orientation video and a positive perception of library usefulness. ${ }^{12}$

Signaling a new era in video tutorial development, Nadaleen Templeman-Kluit and Ethan Ehrenberg's 2003 paper on streaming desktop video capture shared how a tutorial was created and compared screencasting software. ${ }^{13}$ Screencasts, being easy to produce and effective for online instruction, represent a significant portion of the literature on visual tutorials. Maribeth Slebodnik and Catherine Fraser Riehle provided an excellent overview of each element involved in creating animated tutorials, from selecting software to options for evaluation. ${ }^{14}$ Major themes in screencasting include online instruction for distance learners (Daniel Yi Xiao, Barbara Pietraszewski, and Susan Goodwin; ${ }^{15}$ James Waston; ${ }^{16}$ Elaine Peterson; ${ }^{17}$ and Paul Betty ${ }^{18}$ ) as well as software comparison and best practices (Christopher Cox; ${ }^{19}$ Kathleen Carlson; ${ }^{20}$ and Laurie Charnigo ${ }^{21}$ ). Librarians at the University of Colorado at Denver described the use of screencasts across multiple departments, advocating less for perfection and more for quick responses to information needs. ${ }^{22}$ Most recently, articles have examined the use of screencasts beyond user tutorials. For example, Xan Arch noted the use of screencasts to train tech- 
nical services staff ${ }^{23}$ while Allison Carr and Pearl Ly suggested that ad hoc screencasts can enhance reference interactions by providing patrons with directions for complicated searches. ${ }^{24}$

It was not until a few years after screencasts became popular that digital video made a significant appearance in the library literature. One of the first articles to address online instructional tutorials is Karmen Crowther and Alan Wallace's description of the University of Tennessee's streaming video library orientation. ${ }^{25}$ Beginning with this article, many studies have focused on video creation, providing guidelines for successful tutorials. Marketing music library services via short "infomercials" is the subject of Nathalie Hristov and Alan Wallace's 2006 paper. ${ }^{26}$ Diane Mizrachi and Jaclyn Bedoya take a similar approach in utilizing short videos to inform users about library services, collaborating with the student-run campus television team to create commercials that were broadcast on the Office of Residential Life television station and streamed on the library's Web site. ${ }^{27}$ Another method of creating video for student engagement is described by Ramona Islam and Leslie Porter, who produced a brief movie for their library orientation that allowed students to vote on the characters' next actions by using clickers, resulting in a "choose your own adventure" video. ${ }^{28}$ Librarians at Miami University in Ohio made certain that the videos they created became widely available by publishing them in Blackboard and iTunes U. ${ }^{29}$ Like other authors, Lauren Pressley recognized the need to interest students accustomed to "short messages and multimedia." 30 To meet this challenge, North Carolina State University Libraries developed a set of videos on such topics as "Peer-Review in Five Minutes," and "Wikipedia: Beneath the Surface," which are available for use by other libraries. This suite of videos contributes to a burgeoning effort that aims to reduce duplication of efforts in tutorial development. Repositories such as ANimated Tutorial Sharing Project (ANTS), ${ }^{31}$ PeerReviewed Instructional Materials Online Database (PRIMO), ${ }^{32}$ and Multimedia Educational Resource for Learning and Online Teaching (MERLOT) ${ }^{33}$ allow visitors to search for tutorials, download the desired learning object in different file formats, and use them at their own institution.

While no articles surveying video tutorials exist at the time of this study, a small number of authors have surveyed static tutorials. Nancy Dewald was first to systematically evaluate tutorial content, applying principles for effective library instruction to twenty online tutorials. ${ }^{34}$ In 2005 Paul Hrycaj updated Dewald's study to include Association of Research Libraries (ARL) institutions, finding increased active learning components among the tutorials reviewed. ${ }^{35}$ Marta SomozaFernandez and Ernest Abadal assessed nearly two hundred online tutorials created by academic libraries, concluding that most tutorials had noticeable gaps in user evaluation and clear indication of the learning objectives. ${ }^{36}$ Most recently, Sharon Yang found that one-third of one hundred academic libraries developed tutorials in a variety of formats. ${ }^{37}$

Although many articles assist librarians in creating their own tutorials by sharing their expertise or successful initiatives, and several evaluate static Web tutorials, no studies have examined the availability and quality of video tutorials in libraries. Perhaps more surprising is that no studies have focused on tutorials within art libraries, the setting where instructional video most closely aligns with the learning style of their patrons.

\section{Methodology}

From the online list of National Association of Schools of Art and Design (NASAD) member institutions, the author identified Web sites for 305 schools, colleges, and universities in the United States. Ten institutions were found to be duplicate listings, such as universities with programs in Art/ Design and Architecture listed as separate NASAD members. Additionally, library Web sites for five institutions could not be located, bringing the total sample to 290 institutions. The library Web site of each institution was searched for "video," "tutorial," and "screencast," as well as browsed to determine the availability of video tutorials. When appropriate, the author sought a branch library Web site for an institution's art library. If a given library used LibGuides, ${ }^{38}$ a proprietary service for creating subject guides, the LibGuides were searched and browsed separately. Searching was conducted from December 2009 to January 2010.

After locating all possible tutorials, the author evaluated the videos according to a number of criteria, including topic, content, usability, design quality, image quality, duration, output, and software. Each applicable criterion was rated on a scale of zero to five, with zero being extremely poor and five being exceptional. The criteria were identified through best practices in the literature for online instruction (in particular, Susan Sharpless Smith, ${ }^{39}$ Joanne Oud, ${ }^{40}$ and Barbara Blummer and Olga Kritskaya ${ }^{41}$ ) as well as preliminary tutorial viewings, with the goal of selecting measures that review each element of a video that contributes to its success. Because the intent of this study is to evaluate tutorials created by libraries for their patrons, tutorials that linked to another library or vendor were recorded but not assessed. The Appendix contains the complete rubric used to evaluate the tutorials.

Limitations of the study include the fact that course-integrated or password-protected tutorials are not included. The inability to access tutorials behind password protection or embedded in courseware may have resulted in fewer videos located. Additionally, this study includes tutorials published by a given art library's larger system. These tutorials support the needs of art students, and in most cases the art library would not seek to replicate work done by the larger institution.

\section{Results}

A total of 1,300 tutorials were located after searching 290 library Web sites. The findings show that 48.3 percent of libraries (140) included one or more instructional videos on their Web sites, while 51.7 percent (150) did not offer video. Screencasting was the foremost video type and presentation format. Tutorial topics varied widely across sixteen categories, from 20.4 percent of videos addressing specific database searches to 1.6 percent in quizzes and class instruction. The complete findings are detailed below in four sections: Video Tutorial Types, Qualities, Presentation, and Topics.

\section{Video Tutorial Types}

The primary types of multimedia tutorials offered by academic art libraries include screencasts, live video, anima- 
tion, and videos created by other libraries or vendors. Among the 1,300 videos, 82.3 percent $(1,070)$ were created by the home institution, and 17.7 percent of tutorials (230) were produced by a third party, most commonly as a link to a vendor or another library's video.

Screencasts, defined as a screen-recording or series of nonanimated slides created with screencasting software, were by far the most frequent type of video offered, comprising 72.7 percent (778) of the 1,070 original videos. Live videos consisted of 25.3 percent of the total, with most live videos taking the form of footage recorded with a digital camera. Animated videos comprised 2 percent of all tutorials. Tutorials created by third parties, being outside the scope of this study for individual evaluation, are included as a separate group. Figure 1 compares the frequency of each of the four major categories.

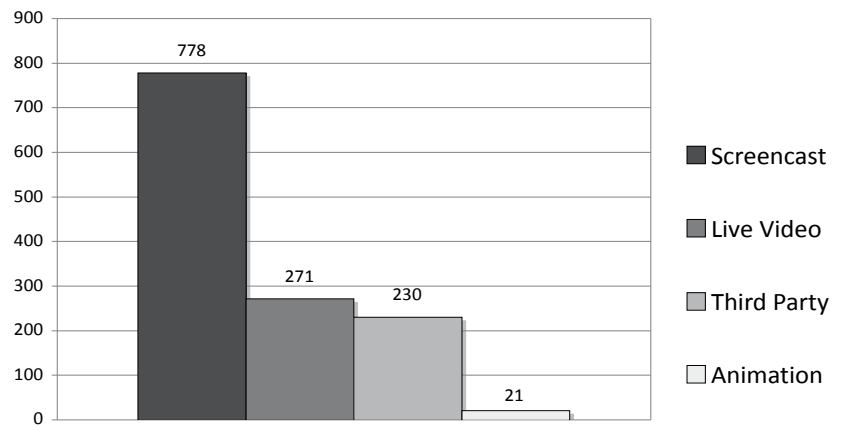

Figure 1. Video Tutorials by Type

\section{Video Tutorial Qualities}

For every tutorial type used by each institution, several factors important to the value of videos as instructional objects were evaluated according to the criteria listed in the Appendix. Each quality was rated on a scale of zero to five. The findings indicate that library tutorials performed best in the categories of design quality and video quality, and least well in the areas of content and audio quality. While the ratings for each criterion had an average score of approximately three, a closer reading indicates the need for improvement in specific areas.

Content addresses the sections, organization, and integration of video tutorials. For example, factors such as whether a video contains an introduction and a closing, whether the content and learning objectives are stated, and the integration of audio and visual information are all evaluated. A total of 148 tutorial types were studied, as eight libraries offered more than one tutorial type (i.e., screencasts as well as live videos). The average rating for content was 2.84 , slightly below the average rating (three). Many videos lacked a content description and information about contacting a librarian for further assistance, resulting in a lower score.

Usability evaluates whether a tutorial provides options for controlling playback, minimizes the use of uncommon file formats, and is easily viewable. In essence, this quality asks whether the video is simple to access and operate. Videos offered in multiple formats, such as both Flash and QuickTime, scored higher. Closed captioning is another feature occasion- ally offered that improves usability. There were 148 tutorial types found to have an average score of 2.91. The usability ratings were narrowly dispersed. Only one video had a rating of zero (extremely poor), while two videos were rated as five (exceptional).

Design Quality asks whether the visual information is presented in an appealing and effectual manner. Because many video tutorials were screencasts and did not contain significant design choices by the video's creator, this criterion applied to only thirty-four tutorial types from thirty-four institutions. When tutorial authors made an effort to incorporate design into videos, whether with an introductory animated sequence or by using iMovie to add a cinematic effect, the overall viewing experience was enhanced. This is evidenced by the average rating of 3.47, the highest rating for any of the six criteria.

Video Quality refers to the quality of the image, focusing on pixilation, appropriate size, and other factors related to viewing the tutorial. The most common issue for videos was a loss of image quality due to compression, as frequently occurs when an already compressed video is uploaded to YouTube. Options for viewing videos of various definitions for different Internet connections received favorable scores. The average Video Quality rating was 3.09.

Audio Quality examines the sound characteristics of a video's narrator, music, or sound effects. While the audio of many tutorials was easy to comprehend, other videos contained background noise that detracted from the overall sound quality. A significant number of videos included either sound effects for "clicks" or "typing," or had no sound at all, resulting in the considerably lower average score of 2.71 for Audio Quality. The average ratings of the five qualities are compared in Figure 2.

The Duration of each instructional video was recorded and rounded to the nearest half-minute. The average length among all videos at a given library was then calculated. If a library

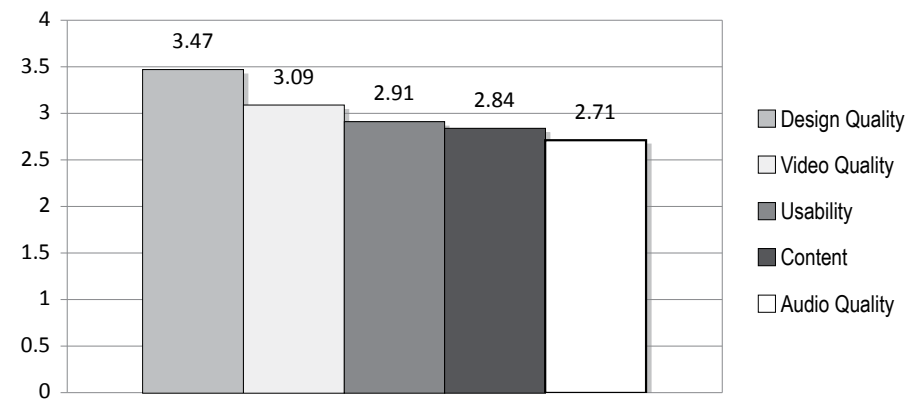

Figure 2. Video Tutorial Quality Ratings

left the duration of a video unnoted, the video was watched in its entirety to determine its length. The findings reveal the average video to be 4.01 minutes, with twenty minutes as the maximum duration and thirty seconds as the minimum. 
Video Tutorial Presentation

The way in which an instructional video is presented to users significantly impacts whether or not the video will be viewed. Videos embedded within a Web page require fewer clicks to view than a video that is linked to a separate page, thereby reducing barriers to access. To determine how videos were offered, the presentation format and output for each video type were recorded and summarized.

Screencasting software was the most popular presentation format, used by a total of 66.2 percent of libraries (ninetyeight). TechSmith's Camtasia was the most frequently used screencasting software at 31.1 percent (forty-six), with Adobe's Captivate software at 29.7 percent (forty-four). Other screencasting applications included TechSmith's Jing at 3.4 percent (five) and three other programs, each at 0.7 percent. YouTube was the most common choice for presenting live videos and occasionally screencasts, with a total of thirty-two institutions (21.6 percent) hosting tutorials on the ubiquitous video-sharing Web site. Four institutions, or 2.7 percent, used Blip.tv. Finally, self-hosted animated videos comprised 6.1 percent (nine) of the total. The presentation format of five libraries could not be determined.

The software used to create a video is important, but it does not tell the entire story. Several output types were encountered while locating videos, including tutorials presented via a library Web site, in LibGuides, or linked to a third party's Web site. If developed by the home institution, it was noted whether tutorials were embedded in a Web page or linked to a separate stand-alone page. The most frequent means of tutorial output was a link to a separate Web page containing the tutorial, with ninety-five of 192 tutorial types (49.5 percent) presented in this mode. Thirty-four institutions (17.7 percent) linked to a third party's Web site, and 10.9 percent (twenty-one) linked directly to their videos in YouTube. Embedding videos was not employed nearly as often as direct links; only twentyfour institutions (12.1 percent) embedded instructional videos in their Web sites. Twelve institutions (6.1 percent) chose to embed tutorials in LibGuides, while 3 percent linked directly to tutorials.

\section{Video Tutorial Topics}

Sixteen distinct topics emerged while viewing video tutorials. A high percentage of tutorials addressed databasespecific searching and navigation, followed by general search strategies/using the catalog and general research assistance. Database searching made up 63 percent of third party tutorials; not surprisingly, more than half of these linked to vendorproduced videos on utilizing their products. An average of 29.5 libraries developed videos for each topic. Furthermore, each library created an average of 2.6 tutorials. Table 1 summarizes video tutorial topics findings.
Table 1. Video Tutorial Topics

\begin{tabular}{|c|c|c|c|c|}
\hline Topic & $\begin{array}{l}\text { Number } \\
\text { of } \\
\text { original } \\
\text { tutorials }\end{array}$ & $\begin{array}{l}\text { Number } \\
\text { of third } \\
\text { party } \\
\text { tutorials }\end{array}$ & $\begin{array}{c}\text { Total } \\
\text { number } \\
\text { of } \\
\text { tutorials }\end{array}$ & $\begin{array}{l}\% \text { of } \\
\text { total }\end{array}$ \\
\hline $\begin{array}{l}\text { Database } \\
\text { searching }\end{array}$ & 120 & 145 & 265 & 20.4 \\
\hline $\begin{array}{l}\text { Search strat- } \\
\text { egies/using } \\
\text { catalog }\end{array}$ & 138 & 18 & 156 & 12 \\
\hline $\begin{array}{l}\text { Research } \\
\text { assistance }\end{array}$ & 85 & 19 & 104 & 8 \\
\hline $\begin{array}{l}\text { Library } \\
\text { services }\end{array}$ & 103 & 0 & 103 & 7.9 \\
\hline Citations & 85 & 17 & 102 & 7.8 \\
\hline $\begin{array}{l}\text { Using } \\
\text { library Web } \\
\text { site }\end{array}$ & 97 & 0 & 97 & 7.5 \\
\hline $\begin{array}{l}\text { Finding } \\
\text { articles }\end{array}$ & 74 & 2 & 76 & 5.9 \\
\hline $\begin{array}{l}\text { Finding } \\
\text { books }\end{array}$ & 58 & 0 & 58 & 4.5 \\
\hline $\begin{array}{l}\text { Identifying } \\
\text { appropriate } \\
\text { resources }\end{array}$ & 45 & 13 & 58 & 4.5 \\
\hline $\begin{array}{l}\text { Library } \\
\text { tour/orien- } \\
\text { tation }\end{array}$ & 53 & 0 & 53 & 4.1 \\
\hline Promotional & 42 & 0 & 42 & 3.2 \\
\hline Other & 42 & 0 & 42 & 3.2 \\
\hline $\begin{array}{l}\text { Using soft- } \\
\text { ware/course } \\
\text { management } \\
\text { system }\end{array}$ & 29 & 9 & 38 & 2.9 \\
\hline $\begin{array}{l}\text { Collections } \\
\text { description }\end{array}$ & 36 & 0 & 36 & 2.8 \\
\hline Plagiarism & 18 & 7 & 25 & 1.9 \\
\hline $\begin{array}{l}\text { Finding } \\
\text { other mate- } \\
\text { rials }\end{array}$ & 24 & 0 & 24 & 1.8 \\
\hline Quizzes & 21 & 0 & 21 & 1.6 \\
\hline Total & 1,070 & 230 & 1,300 & 100 \\
\hline
\end{tabular}

Many tutorials on the topic of Citations instructed users on particular citation styles. Instructional videos on using citation programs such as RefWorks were included in this 
category, resulting in a higher number of tutorials. Videos on citations comprised 7.8 percent of the total (108) with seventeen directing users to tutorials produced by third parties.

Collections description denotes videos endorsing particular library collections. Tutorials in this category describe a specific collection or department but do not provide a general tour or overview of the library. Thirty-six ( 2.8 percent) of the videos address this topic.

Finding articles was a topic found in a range of institutions, with thirty-eight libraries developing tutorials to assist users in this area. These videos frequently took the form of screencasts demonstrating how to access databases from the library's homepage. Seventy-six (5.9 percent) of the tutorials addressed finding articles, with two tutorials by third parties.

Instructional videos on Finding books were not as prevalent as those for finding articles. Fifty-eight institutions (4.5 percent) offered tutorials on locating books. Tutorials such as "Finding books using the library catalog" that could be classified as either "finding books" or "using the catalog" were included in this category, as finding books was presented as the objective. Videos on navigating the library stacks and using call numbers were also grouped under this subject.

Finding other materials related to locating items such as DVDs, audio-visual equipment, or other materials not classified as articles or books. Instruction in this area, as with finding articles and books, typically consisted of demonstrating how to determine the availability of these materials via the library Web site. Twenty-four libraries offered online video instruction on this topic, which comprised 1.8 percent of all tutorials.

Identifying appropriate resources included tutorials that assisted patrons in selecting appropriate databases, explaining how to identify scholarly materials, and providing guidelines for evaluating Web sites. For example, one animated video explained how Wikipedia is created and when it is appropriate as a research tool. Fifty-eight (4.5 percent) of the videos instructed patrons in this area, with thirteen developed by another library.

Library services tutorials addressed topics such as interlibrary loan, group study rooms, printing or scanning documents, and other physical or virtual services. Reference services were included under the research assistance category. Exemplary videos in this area included a series of scripted one-minute videos describing a single library service. Library services consisted of 7.9 percent of tutorials (103).

Library tour/orientation videos accounted for 4.1 percent of tutorials (fifty-three). Appropriately, a majority of online library tours featured video footage of library buildings as opposed to screencasts. The most common forms of library orientation videos were narrated tours of the building or brief descriptions of the library's highlights. One creative approach to this common topic was a video presented as a "survival guide" for students that contained "library secrets."

Plagiarism tutorials were infrequent, consisting of 1.9 percent of all videos (twenty-five). Seven of these tutorials linked to other libraries. A majority of videos on this topic defined plagiarism, illustrated how to avoid plagiarism, and emphasized its damaging effect on one's academic career.

Promotional videos included student and faculty testimonials to the library's value, library staff profiles, and other outreach videos designed to promote the organization in a broader sense. Forty-two tutorials (3.2 percent) endorsed the library though promotional videos. One instance of excellent promotional videos uses Apple Computer's popular "Get a Mac" advertisements as inspiration for a four-episode series titled "Librarian vs. Stereotype." In each episode two people, one of whom represents a Librarian and the other a Librarian Stereotype, confront librarian misconceptions in a humorous manner.

Very few libraries presented Quizzes $\mathcal{E}$ class instruction tutorials, which accounted for 1.6 percent of all videos (twentyone). Quizzes tested user retention of related instructional videos and were included separately from their associated topic. Class instruction videos conveyed information about course assignments to students.

Research assistance accounted for 8 percent of videos (104), with nineteen videos created by third parties. Tutorials on conducting a literature review, contacting a reference librarian, and writing a research paper are examples. A majority of tutorials addressed Database searching. Whereas other tutorials instructed users in general search strategies or searching the catalog, videos in this category provided assistance in using a particular database. Examples include "Find articles using EBSCO" and "Searching JSTOR." Third parties created more than half of the tutorials. Database searching represented 20.4 percent of tutorials (265).

Another frequently encountered subject was that of Search strategies/using the catalog. Screencasts were a very popular presentation format for this topic. Videos addressed Boolean Searching, Using Advanced Search in the Catalog, and related subjects. Search strategies or catalog use were taught in 12 percent of tutorials (156). Using library Web site tutorials most frequently took the form of instruction on performing a task using the library Web site, such as "How to View Your Library Account," or "Using Journal Finder." Other videos gave users a brief introduction to the library Web site. Using Library Web site tutorials represented 7.5 percent of the sample (ninetyseven).

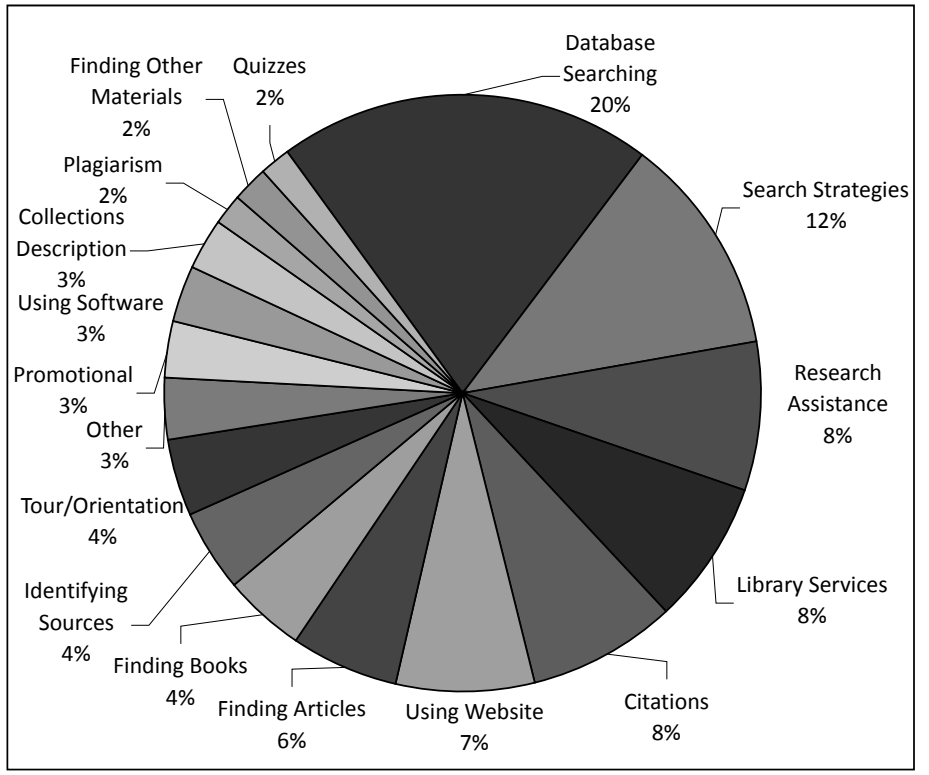

Figure 3. Video Tutorial Topic Frequency 
Tutorials on Using software/course management system often demonstrated a specific function within popular software products. Examples of tutorials on this topic include "Editing Video with Windows Movie Maker," and "Discussion Boards in Blackboard." Thirty eight tutorials (2.9 percent) provided instruction on this subject.

As demonstrated by these sixteen categories, video tutorials encompass a wide range of subjects. Videos not represented by other topics were classified as Other, which represent 3.2 percent of the total sample (forty-two). One illustration of this category includes footage from the library archives presented on YouTube, documenting momentous occasions in the university's history. Figure 3 shows the frequency of video tutorial topics.

\section{Conclusion}

Academic libraries supporting students in the visual arts appear to recognize the need to provide engaging online instruction. Although the answer to the question "Are academic and art school libraries offering instructional videos?" is not a resounding yes, nearly half of the 290 institutions provide video tutorials, and an increase in that number is highly likely. Further, the tutorials were generally of brief duration, spoke to a broad range of topics, and demonstrated an understanding of design qualities. Few tutorials addressed the needs of art students explicitly and instead taught non-discipline-specific skills appropriate for a wider audience. Tutorials specific to art and design studies included topics similar to general library tutorials, but tailored to their audience's needs. Some examples include ARTstor search techniques, an overview of image resources available at one institution, and an introduction to services offered by a branch art library.

Video Tutorial Types, consisting of screencasts, live video, animation, and third party tutorials, were primarily screencasts. The overwhelming popularity of screencasts as a method for online instruction may be due to their ease of production, particularly when compared to the myriad possibilities presented while developing a live video or animation. Because screencasting software offers a standardized format for content and publication, it presents a consistent, though perhaps less personable, learning experience. Another point of consideration is that over 80 percent of tutorials were created by the home institution. This commendable effort suggests that libraries are attempting to address the needs of their particular populations. At the same time, it is important to be aware of options for tutorial creation outside of one's institution. Videos developed by third parties can be an excellent way to avoid the proverbial reinvention of the wheel.

Video Tutorial Presentation results show clear preferences for screencasting software and Web sites to share videos. The most popular screencasting programs were Camtasia and Captivate. More than 60 percent of all tutorials were created using one of these two programs. Surprisingly, few libraries embedded instructional video in their Web sites. In some cases providing links to tutorials may be a choice made to reduce clutter on a Web page, but the advantages of embedding video, including allowing viewers to remain on the same Web page and providing a visual preview of the content, should not be overlooked.
Video Tutorial Topics reveal an emphasis on videos demonstrating use of library resources. Topics such as database searching, locating books or articles, and search strategies are well represented and are highly suitable for online instruction due to their systematic step-by-step approach. However, seeking other topics is recommended to provide additional engagement. A set of screencasts providing database search strategies may be useful, yet they lack the curiosity factor that may lead a user to click on a tutorial in the first place. As demonstrated by the most engaging videos from the sample, a range of topics combined with a small dose of creativity can go a long way towards piquing patron interest.

For art librarians seeking to develop video tutorials of their own, the five tips below may assist those beginning the process:

- Determine whether an online video tutorial is the most appropriate method for your purpose, according to your audience and learning objectives.

- Use a storyboard and/or script to plan your video in advance.

- Include both audio and text to accommodate multiple learning styles.

- Increase accessibility by including closed captioning, slide notes, and a menu whenever possible.

- Have your tutorial reviewed by a colleague before its completion, and solicit user feedback to assess the completed tutorial.

In addition to these tips, Slebodnik and Riehle's guide mentioned above covers all aspects of screencast creation. The Appendix also contains questions that may be considered to improve video tutorials.

At this juncture, art libraries already offering tutorials may need to focus on providing engaging content. More inventive topics can be addressed, beyond searching and database instruction. Videos can incorporate live footage along with animation, beyond recordings of computer screens that lack a personalized element. Students can be recruited to produce and contribute to videos. The goal is to continue to deliver instruction that both informs and engages. Online video is a popular medium that will only become more entrenched in daily Web activities. Now is the time for academic art libraries to take advantage of video's many possibilities for serving their users.

\section{Notes}

1. Gail Wood, Lorraine Melita, and Marc Wildman, “Lights! Cameras! Action!: Digital Media Equipment and the Academic Library," Journal of Academic Librarianship 30, no. 5 (2004): 410-15.

2. Susan L. Silver and Lisa T. Nickel, "Are Online Tutorials Effective? A Comparison of Online and Classroom Library Instruction Methods," Research Strategies 20, no. 4 (2005): 389-96; Penny Beile and David Boote, "Does the Medium Matter? A Comparison of a Web-based Tutorial with Face-to-Face Library Instruction on Distance Education Students' Self-Efficacy Levels and Learning Outcomes," Research Strategies 20, no. 1-2 (2005): 57-68; Elizabeth Blakesley Lindsay, Lara Cummings, Corey M. Johnson, and B. Jane Scales, "If You Build It, Will They Learn? Assessing Online Information Literacy Tutorials," College $\mathcal{E}$ Research Libraries 67, no. 5 (2006): 429-45; Elizabeth Kraemer, 
Shawn Lombardo, and Frank Lepkowski, "The Librarian, the Machine, or a Little of Both: A Comparative Study of Three Information Literacy Pedagogies at Oakland University," College \& Research Libraries 68, no. 4 (2007): 330-42.

3. Li Zhang, Erin M. Watson, and Laura Banfield, "The Efficacy of Computer-Assisted Instruction Versus Face-to-Face Instruction in Academic Libraries: A Systematic Review," Journal of Academic Librarianship 33, no. 4 (2007): 478-84.

4. Nadaleen Tempelman-Kluit, "Multimedia Learning Theories and Online Instruction," College E Research Libraries 64, no. 4 (2006): 364-69.

5. John L. Lolley, "Videotape Programs," Drexel Library Quarterly 16, no. 1 (1980): 83-102.

6. Gertrude N. Jacobson and Michael J. Albright, "Motivation via Videotape: Key to Undergraduate Library Instruction in the Research Library," Journal of Academic Librarianship 9, no. 5 (1983): 270-75.

7. Barbara A. Kautz, "The Evolution of a New Library Instruction Concept: Interactive Video," Research Strategies 6, no. 3 (1988): 109-17; Nancy French and Julene H. Butler, "Quiet on the Set: Library Instruction Goes Video," Wilson Library Bulletin 63, no. 4 (1988): 42-44.

8. Jean Smith, "Teaching Research Skills Using Video: An Undergraduate Library Approach," Reference Services Review 16, no. 1-2 (1988): 109-114.

9. James Shedlock and Edward W. Tawyea, "Library Orientation On Videotape: Production Planning and Administrative Support," Medical Reference Services Quarterly 8, no. 2 (1989): 15-24.

10. Ann Taylor Blauer, "Librarian, Clone Thyself! Using a Video to Promote Your Library Service," in The Seventh Off-Campus Library Services Conference Proceedings: San Diego, California, October 25-27, 1995, ed. Carol Jacobs, 17-23 (Mount Pleasant, MI: Central Michigan University, 1995).

11. Daren Callahan, “'Two Thumbs Up!' Library Applications for Video Technology," Information Technology and Libraries 14, no. 3 (1995): 173-76.

12. Eileen Wakiji and Joy Thomas, "MTV to the Rescue: Changing Library Attitudes Through Video," College \& Research Libraries 58, no. 3 (May 1997): 211-16.

13. Nadaleen Tempelman-Kluit and Ethan Ehrenberg, "Library Instruction and Online Tutorials: Developing Best Practices for Streaming Desktop Video Capture," Feliciter 49, no. 2 (2003): 89-90.

14. Maribeth Slebodnik and Catherine Fraser Riehle, "Creating Online Tutorials at Your Libraries: Software Choices and Practical Implications," Reference E User Services Quarterly 49, no. 1 (2009): 33-37.

15. Daniel Yi Xiao, Barbara A. Pietraszewski, and Susan P. Goodwin, "Full Stream Ahead: Database Instruction Through Online Videos," Library Hi Tech 22, no. 4 (2004): 366-74.

16. James Watson, "Going Beyond Screen Captures: Integrating Video Screen Recording into Your Library Instruction Program," Feliciter 50, no. 2 (2004): 66-67.

17. Elaine Peterson, "Incorporating Screencasts in Online Teaching," International Review of Research in Open and Distance Learning 8, no. 3 (2007): 1-4.

18. Paul Betty, "Creation, Management, and Assessment of Library Screencasts: The Regis Libraries Animated Tutorials Project," Journal of Library Administration 48, no. 3/4 (2008): 295-315.
19. Christopher Cox, "From Cameras to Camtasia: Streaming Media Without the Stress," Internet Reference Services Quarterly 9, no. 3-4 (2005): 193-200.

20. Kathleen Carlson, "Delivering Information to Students 24/7 with Camtasia," Information Technology $\mathcal{E}$ Libraries 28, no. 3 (2009): 154-56.

21. Laurie Charnigo, "Lights! Camera! Action! Producing Library Instruction Video Tutorials Using Camtasia," Journal of Library E Information Services in Distance Learning 3, no. 1 (2009): 23-30.

22. Margaret Brown-Sica, Karen Sobel, and Denise Pan, "Learning for All: Teaching Students, Faculty, and Staff with Screencasting," Public Services Quarterly 5, no. 2 (2009): 81-97.

23. Xan Arch, "Screencasting for Acquisitions," Against the Grain 20, no. 6 (2008): 66.

24. Allison Carr and Pearl Ly, "'More Than Words': Screencasting as a Reference Tool," Reference Services Review 37, no. 4 (2009): 408-20.

25. Karmen Crowther and Alan Wallace, "Delivering Video-Streamed Library Orientation on the Web: Technology for the Educational Setting," College E Research Libraries News 62, no. 3 (2001): 280-85.

26. Nathalie M. Hristov and Alan Wallace, "Marketing Music Library Services Through Video Infomercial and Resource Guides: A Case Study from the University of Tennessee George F. DeVine Music Library," Music Reference Services Quarterly 10, no. 3-4 (2006/2007): 1-24.

27. Diane Mizrachi and Jaclyn Bedoya, "LITE Bites: Broadcasting Bite-Sized Library Instruction," Reference Services Review 35, no. 2 (2007): 249-56.

28. Ramona Islam and Leslie Porter, "Perseverance and Play: Making a Movie for the YouTube Generation," Urban Library Journal 15, no. 1 (2008).

29. Jason P. Michel, Susan Hurst, and Andrew Revelle, "Vodcasting, iTunes U, and Faculty Collaboration," E-JASL: The Electronic Journal of Academic and Special Librarianship, http:// southernlibrarianship.icaap.org/content/v10n01/michel j01. html, 10, no. 1 (2009).

30. Lauren Pressley, "Using Videos to Reach Site Visitors: A Toolkit for Today's Student," Computers in Libraries 28, no. 6 (2008): 18-22.

31. ANimated Tutorial Sharing Project (ANTS), http:// ants.wetpaint.com (accessed June 1, 2010).

32. Peer-Reviewed Instructional Materials Online Database (PRIMO), http://www.ala.org/apps/primo/public/search.cfm (accessed June 1, 2010).

33. Multimedia Educational Resource for Learning and Online Teaching (MERLOT), http://www.merlot.org/merlot/ materials.htm (accessed June 1, 2010).

34. Nancy H. Dewald, "Transporting Good Library Instruction Practices into the Web Environment: An Analysis of Online Tutorials," The Journal of Academic Librarianship 25, no. 1 (1999): 26-31.

35. Paul L. Hrycaj, "Elements of Active Learning in the Online Tutorials of ARL Members," Reference Services Review 33, no. 2 (2005): 210-18.

36. Marta Somoza-Fernandez and Ernest Abadal, "Analysis of Web-Based Tutorials Created by Academic Libraries," Journal of Academic Librarianship 35, no. 2 (2009): 126-31. 
37. Sharon Yang, "Information Literacy Online Tutorials: An Introduction to Rationale and Technological Tools in Tutorial Creation," The Electronic Library 27, no. 4 (2009): 684-93.

38. LibGuides Community Web Site, http:/ / www. libguides.com.

39. Susan Sharpless Smith, Web-Based Instruction: A Guide for Libraries, $2^{\text {nd }}$ ed. (Chicago: American Library Association, 2006).

40. Joanne Oud, "Guidelines for Effective Online Instruction Using Multimedia Screencasts," Reference Services Review 37, no. 2 (2009): 164-77.

41. Barbara A. Blummer and Olga Kritskaya, "Best Practices for Creating an Online Tutorial: A Literature Review," Journal of Web Librarianship 3, no. 3 (2009): 199-216.

\section{Appendix: Criteria Used to Evaluate Video Tutorials}

- Content

o Does the video contain an introduction noting the author and/or institution?

o Is the content clearly stated and appropriate for the intended audience?

o Are the video's components, such as audio, visuals, and text, well integrated?

o Does the video contain information on obtaining further assistance?

- Usability

o Do viewers have the opportunity to view the content at their own pace?

o Is the video easy to navigate?

o Are technical barriers to access minimized? Barriers may include significant load time, plug-ins required for viewing, or utilizing an uncommon file format.

- Design Quality

o Is the visual information presented in an attractive and effective manner?

o Do the content and design convey attention to detail, such as the absence of grammatical errors and maintenance of the same design elements throughout?

- Video Quality

o Does the video appear pixilated? Was there a significant loss of image quality when it was uploaded?

o Is the video an appropriate size for the screen?

- Audio Quality

o Is the audio easily comprehended and without distortion? o Is unwanted background noise audible?

- Duration

o Is the video brief enough to sustain viewer attention?

o Have large topics been divided into several shorter videos?

- Output

o Is the video embedded on the library Web site where it can be easily viewed?

o Has the video been uploaded to YouTube or another video sharing Web site, increasing the tutorial's visibility?

- Software

o Which recording software was chosen, if appropriate?

- Topic

o Is the mode of presentation appropriate for the subject, or would the topic be better suited to a different format (i.e., a text-based Web page)?

Eamon Tewell, Senior Library Assistant Moore College of Art $\mathcal{E}$ Design, Philadelphia, Pennsylvania, etewell@moore.edu

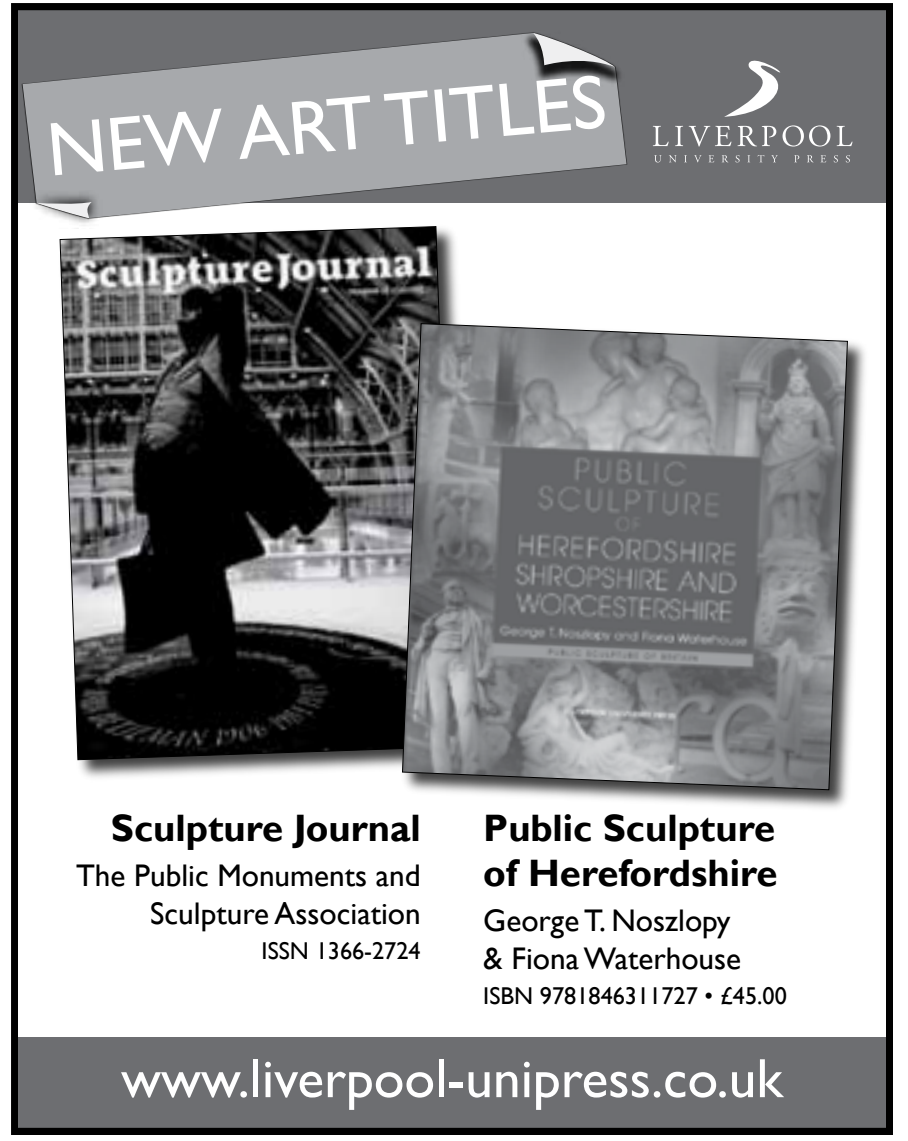

